\title{
Quantitative fluorescent PCR (QF-PCR) for prenatal diagnosis of fetal aneuploidy: Indian experience
}

\author{
Kusagradhi Ghosh $^{1, *}$, Giridharan Appaswamy², Tippabathani Jayakrishna ${ }^{3}$ \\ ${ }^{1}$ Professor, Dept. of Obstetrics and Gynecology, VIMS Kolkata Health University, Kolkata, ${ }^{2}$ Chief Scientific Officer, ${ }^{3}$ Senior \\ Research Associate, Life Cell International Pvt Ltd, Chennai, Tamil Nadu, India
}

*Corresponding Author:

Email: ghoshkusha@gmail.com

Received: $9^{\text {th }}$ May, 2018

Accepted: $29^{\text {th }}$ May, 2018

\begin{abstract}
Introduction: Prenatal diagnosis of fetal chromosomal aneuploidies has traditionally relied on cytogenetics. In recent times QFPCR, to detect the common aneuploidies has been reported. In view of the paucity of Indian experience regarding QF-PCR, we present prospective data from our centre.

Materials and Methods: 572 AF samples were collected from Indian women with singleton pregnancy between 16-20 weeks gestation after counselling. The samples were collected over one year and followed up till delivery. AF was sent for QF-PCR for chromosomes 13, 18, 21 and 23.

Results: There was no procedure related miscarriage, or IUFD. There were seventeen trisomy 21, two 47XXX and one 47XXY. All pregnancies with fetal aneuploidies underwent termination. All 552/572 women with normal report were followed up and none had IUFD or clinical features of trisomy 21 after term or preterm births.

Discussion: The current study stresses the clinical importance of shorter TAT advantage and the slight cost advantage of QFPCR over FISH. The current study shows QF-PCR is a satisfactory method for diagnosing chromosomal aneuploidy from amniotic fluid in screen positive population, has advantages and might be preferred more in near future in place of FISH, especially in the scenario of increasing awareness of biochemical screening for chromosomal aneuploidies in India. However, more such studies with increased numbers and in multiple pregnancies are required. The current study is ongoing and hopefully we will be able to present such data soon.
\end{abstract}

Keywords: QF-PCR, Aneuploidy, Prenatal diagnosis, Segmental duplication.

\section{Introduction}

Chromosomal aneuploidy is the second major category of chromosome aberration where chromosome number is abnormal. An aneuploid is an individual organism whose chromosome number differs from the normal population by part of a chromosome set. Over the last few decades, prenatal diagnosis of fetal chromosomal abnormalities has relied on conventional cytogenetic analysis of cultured amniocytes, chorionic villi, or fetal blood by percutaneous umbilical cord blood. ${ }^{1}$ Since conventional G-banding cytogenetic analysis (Karyotype) is time consuming (2-3 weeks), quantitative methods measuring contributions from individual chromosomes have been used for rapid detection of chromosomal abnormalities. ${ }^{2}$ FISH has been shown to be an accurate method to detect fetal aneuploidy, and commercial kits are available to test for trisomy 13, 18, 21, and sex chromosome aneuploidies. FISH is a routine method used for rapid detection in interphase amniocytes thereby avoiding the need to culture cells. ${ }^{3}$ However, the technique is expensive, complex, labor intensive, requires technical expertise, has limited resolution and prevents the efficient processing of a large number of samples in a diagnostic setting. In the last few years, the clinical validity of a newer technique, ${ }^{4}$ QF-PCR, to detect the common aneuploidies has been reported by a number of investigators. In Indian population QF-PCR has not been extensively used so far. In view of the paucity of Indian experience regarding QF-PCR as a tool for prenatal diagnosis of chromosomal aneuploidies, we present prospective data from our centre in this paper. We hope this adds value and clarify certain doubts regarding this emerging technology and its application in prenatal diagnosis.

\section{Materials and Methods}

This is an ongoing study commencing from 01.11.2017. Prior to that FISH was used for rapid detection of fetal aneuploidy from amniotic fluid.

Study Samples: Pregnant women with clinical recommendation for prenatal diagnosis of trisomy 21 are referred to our center by their Obstetricians. Women carrying singleton pregnancy were included in this study to maintain homogeneity. 572 amniotic fluid (AF) samples were collected from Indian women between 16-20 weeks gestation. The samples were collected between 01.11.2016 and 30.10.2017 (one year) and followed up till delivery. All the participants in the study received genetic counselling with detailed information on the advantages and limitations of the rapid QF-PCR assay and provided written informed consent for amniocentesis and QF-PCR. After proper counselling, $10 \mathrm{ml}$ of amniotic fluid was aspirated under USG guidance using a $23 \mathrm{G}$ spinal needle and sent to Fetomed laboratories, Chennai. India. 
DNA Extraction: Genomic DNA was extracted from uncultured amniotic fluid samples using the QIAamp DNA mini kit (Qiagen). After extraction, the quantity and quality were evaluated by spectrophotometry on a Nanodrop 2000 (Thermo Fisher Scientific). DNA samples from Coriell Life Sciences positive for Trisomy 13, 18, 21 and gonosomal aneuploidies were used as internal control to evaluate the sensitivity and specificity of the assay.

Multiplex SD QF-PCR: The segmental duplication markers used in the study are two similar sequences located on two different chromosomes but different in fragment lengths when co-amplified using a single pair of primers. At least two segmental duplications for each target chromosome was used in the study to detect aneuploidies of chromosome 13, 18, 21 and the gonosomes. ${ }^{5}$ All the PCR primers used in the study were synthesized and purified by HPLC at Genscript, USA.

PCR amplification was performed using Applied Biosystems ${ }^{\circledR}$ Veriti ${ }^{\circledR}$ Dx PCR system in a total reaction volume of $25 \mu \mathrm{L}$ with $20 \mathrm{ng}$ of DNA and $5 \mu \mathrm{M}$ primers (forward and reverse). The reaction is carried out with $95^{\circ} \mathrm{C}$ initial denaturation for $3 \mathrm{~min}$, followed by 28 cycles of $95^{\circ} \mathrm{C}$ for $30 \mathrm{secs}$ (denaturation), $60^{\circ} \mathrm{C}$ for $30 \mathrm{secs}$ (annealing) and $72^{\circ} \mathrm{C}$ for 30 secs (extension) followed by final extension of $72^{\circ} \mathrm{C}$ for $5 \mathrm{~min}$.

Fragment Analysis: Amplification was initially confirmed by a $2 \%$ agarose gel run and the amplification products were further subjected to fragment analysis by capillary electrophoresis. Approximately $1 \mu \mathrm{L}$ of PCR product and was mixed with $24 \mu 1$ of formamide and $1 \mu 1$ of size standard (Applied Biosystem). The mixture was denatured at $95^{\circ} \mathrm{C}$ for $3 \mathrm{~min}$ and placed on ice to prevent the reannealing until further analysis. Fragment analysis was performed using ABI Genetic analyser 3500 Dx. Gene mapper ID software V3.2 was used for the data analysis of product length and volume amplification.

Relative fluorescence intensity as measured by peak height was calculated for the target and reference chromosome specific amplicon. The obtained ratios were plotted against a population specific normalized value to obtain the final ratio of each target chromosome. The values are $\leq 1.25$ for euploid and $\leq$ 1.25-1.5 for trisomic condition indicating the presence of an additional copy of the chromosome. ${ }^{6}$

\section{Results}

The participants in this study had a mean maternal age of 32.30 6.53 years and ranged between 21-42 years. Among those $18.8 \%$ were above 35 years older. The indications for amniocentesis are shown in Table 1. Results of total 572 sample were summarized in Table 2 and electrogramme of aneuploidy detection was shown in Fig. 1.

Table 1: Indications for invasive test (amniocentesis) in the study population

\begin{tabular}{|l|c|c|}
\hline \multicolumn{1}{|c|}{ Indications for Amniocentesis } & Number & \% \\
\hline First trimester screen positive (NT, PAPPA, free beta HCG) & 261 & 45.6 \\
\hline Second trimester Quadruple screen positive & 258 & 45.1 \\
\hline Advanced Maternal Age (Biochemical Screening not done) & 40 & 7.0 \\
\hline Previous history of Trisomy 21 baby & 10 & 1.8 \\
\hline Maternal anxiety & 3 & 0.5 \\
\hline Total & $\mathbf{5 7 2}$ & $\mathbf{1 0 0}$ \\
\hline
\end{tabular}

Table 2: Results on QF-PCR analysis of 572 samples and the detection of whole chromosomal abnormalities

\begin{tabular}{|c|c|c|c|c|}
\hline No of Amniocentesis & \multicolumn{3}{|c|}{ Detected Chromosomal Aneuploidy } & Without Aneuploidy \\
\hline & Trisomy 21 & XXX & XXY & \\
\hline 572 & 17 & 2 & 1 & 552 \\
\hline
\end{tabular}

Marker 13/9

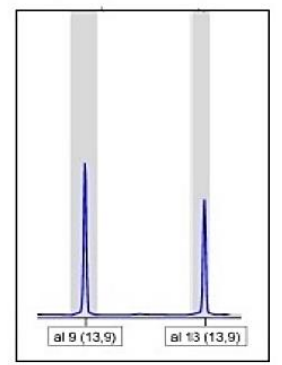

Unaffected

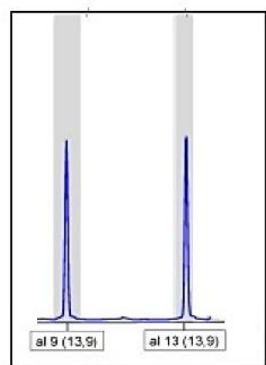

Trisomy 13

Marker 13/11

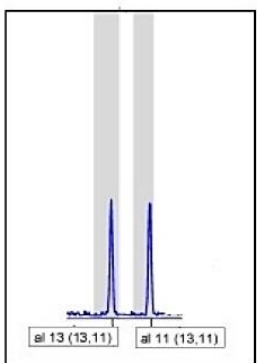

Unaffected

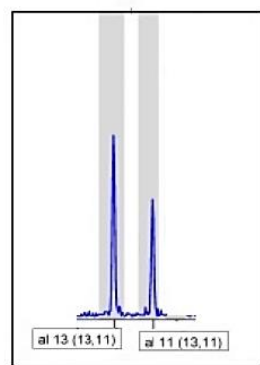

Trisomy 13

Fig. 1: Electrogram for aneuploidy detection of trisomy 13 
Marker 18/10

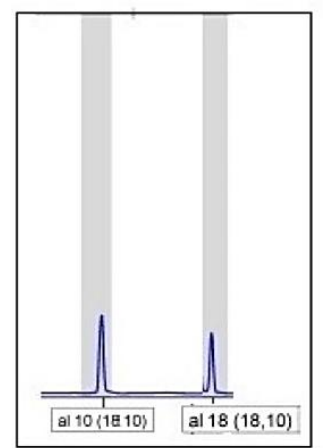

Unaffected

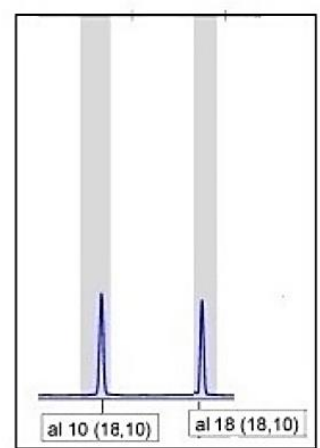

Trisomy 18
Marker 18/1

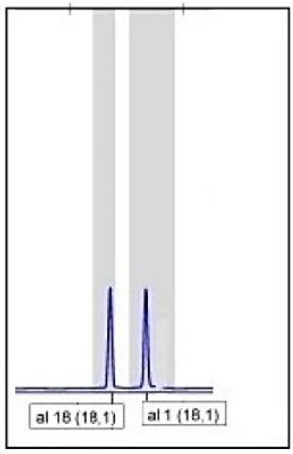

Unaffected

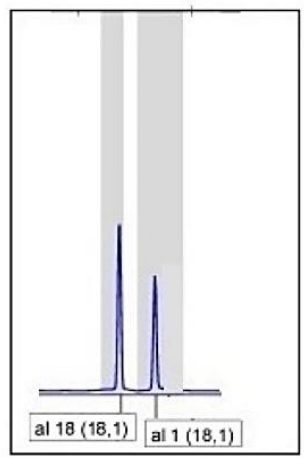

Trisomy 18

Fig. 2: Electrogram for aneuploidy detection of trisomy 18

Marker 21/11

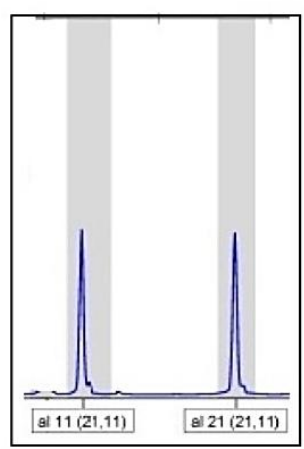

Unaffected

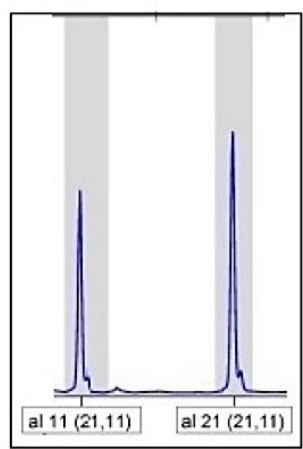

Trisomy 21

Fig. 3: Electrogram for aneuploidy detection of trisomy 21

Validation: The results of 39 random samples tested negative by SD QF-PCR for whole chromosomal aneuploidies were subjected to karyotype analysis to identify any false negative results. All 20 samples tested positive by SD QF-PCR was further confirmed positive by an Elucigene QSTR kit indicating a high sensitivity and specificity of the SD QF-PCR (data not shown). Moreover, we had also attempted to correlate the results for a few samples with maternal cell contamination in comparison to the uncontaminated samples and observed that despite the red blood cell contamination in 5 samples, our assay can be used to successfully when the levels of maternal cell contamination are $<20 \%$ (data not shown).

Follow up: All the subjects in the study population were followed up. There was no procedure related miscarriage. All the 20 fetuses who tested positive for aneuploidy underwent termination of pregnancy. There was no IUFD in the study population. There were 52 deliveries between 29-34 weeks and the rest were delivered after 34 weeks, either vaginally or by LSCS. None of the babies of the 552 women who tested
Marker 21/6

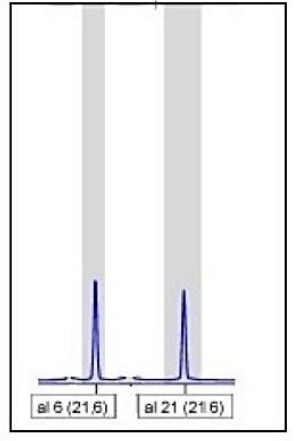

Unaffected

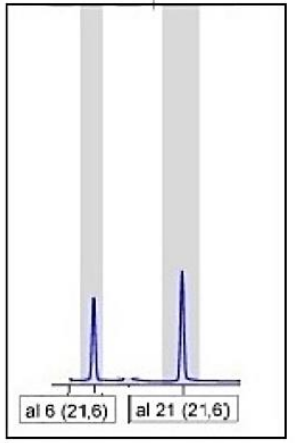

Trisomy 21 normal on QF- PCR had any clinical features of trisomy 21 after term or preterm births.

\section{Discussion}

Screening for Down syndrome is gradually getting popular among pregnant women as well as the caregivers. This is because of increasing awareness and biochemical screening in first or second trimesters. Once women are triaged as 'screen positive' they are referred to fetal medicine centers for subsequent counselling and management. Our study population mainly belonged to the state of West Bengal and adjoining states in India.

All women were thoroughly counselled and option was given for NIPT or amniocentesis. Majority of such women preferred amniocentesis and the rest opted for NIPT. The major reasons for opting for amniocentesis were four: firstly, the higher cost of NIPT, secondly, the longer time required to get the NIPT report (two weeks in NIPT versus four days after amniocentesis), thirdly, the possibility of recall and fourthly, the false negative rate of $0.3 \%$ with NIPT. 572 women who opted for amniocentesis after counselling 
between 01.11.2016 and 30.10.2017 were included in the study and followed up till delivery.

Karyotype is still considered as gold standard as far as cytogenetics is considered. However, it suffers from a major drawback that it takes 2-3 weeks before a report can be generated. Therefore, rapid aneuploidy testing (RAT) procedures have become popular for quicker results. The conventional cytogenetic RAT for chromosomal aneuploidy with amniotic fluid is FISH (fluorescent in situ hybridisation) using fluorescent probes for chromosomes 13. 18. and 21. Of late, there has been interest in molecular genetics like QF-PCR and chromosomal microarray for diagnosis of chromosomal aneuploidy.

QF-PCR is DNA analysis whereas FISH is individual cell analysis through a microscopic and hence labour intensive and may be subjective to human error. A single technician can handle hundreds of QFPCR samples per day due to the high automation capabilities. Therefore, as the sample numbers of amniotic fluid received by genetic laboratory increases, QF-PCR has definite advantage over FISH. ${ }^{7}$

The turnaround time for QF-PCR is quicker by at least 48 hours compared to FISH. 'Screen positive' for trisomy 21 is an emotive issue for the parents as well as the caregivers. In the current study population, during counselling before amniocentesis, it was mentioned that the turnaround time was 2 days less in QF-PCR compared to FISH. Post counselling, all our patients opted for QF-PCR. The cost for QF-PCR for chromosomes 13, 18. 21 and 23 was slightly less than FISH for the same chromosomes. But the difference in the cost was not the major determining factor in this cohort. After amniocentesis, the wait for the result is stressful for everybody. A quicker turnaround time is clinically very relevant in this scenario.

The current study stresses the importance of shorter TAT advantage of QF-PCR over FISH. The FISH probes are relatively costly and only limited probes can be used. DNA based test like QF-PCR has unlimited opportunity for testing for other disorders as well which may be of relevance in some cases. There is paucity of data using QF-PCR for diagnosis of chromosomal anomalies from amniotic fluid in Indian population. The current study shows that QF-PCR is a satisfactory method for diagnosing chromosomal anomaly from amniotic fluid in screen positive population, has advantages and might be preferred more in near future in place of FISH. However, more such studies with increased numbers and in multiple pregnancies are required. The current study is ongoing and hopefully we will be able to present such data soon.

Overall, considering the clinical demand for the rapid prenatal results, Segmental duplication QF-PCR proves to be a valid to detect whole chromosomal aneuploidies and a method as compared to more laborious molecular cytogenetic techniques such as FISH and prenatal BOBs. Segmental duplication QF-
PCR would also be feasible and inexpensive to perform rapid screening for aneuploidy detection on all prenatal samples with a turnaround time of just 1-2 business days, considering the need for the same in most cases. The speed and apparent reliability of this technique has thus significantly reduced the waiting time for clinicians and anxious prospective parents alike, to understand the risk and confirm that the fetus does not carry any whole chromosomal abnormality of 13,18 , 21 and the gonosomes.

Moraes et al., 2017, ${ }^{8}$ had reported failures in the QF-PCR which might have occurred because of use of STR markers which led the test accuracy to drop to 93\% as compared to karyotyping where $100 \%$ accuracy was observed. Similarly, Srinivasan et al., 2014, ${ }^{9}$ showed that the lack of heterozygosity data lead to the failure of QF-PCR to detect aneuploidies in a north Indian population. In this condition, our results show that the test accuracy of our QF-PCR assay was $100 \%$, since we had used SD markers instead of STR markers. ${ }^{8}$ SD markers were independent on the population whilst STR markers depends on the heterozygosity data. In such cases, the development and validation of an assay becomes difficult.

\section{Conclusion}

Prenatal diagnosis of fetal chromosomal abnormalities has traditionally relied on cytogenetic analysis (karyotype) of amniocytes, chorionic villi, or fetal blood. Since this is time consuming and labor intensive, quantitative methods have been used for rapid detection. FISH has been shown to be an accurate method to detect fetal trisomy 13,18, 21, and sex chromosomes aneuploidy. However, FISH is expensive, complex, labor intensive, requires technical expertise, has limited resolution and processing of a large number of samples poses challenge. In the last few years, the clinical validity of QF-PCR, to detect the common aneuploidies has been reported by a number of investigators. In view of the paucity of Indian experience regarding QF-PCR, we present prospective data from our centre.572 amniotic fluid (AF) samples were collected from Indian women with singleton pregnancy between 16-20 weeks gestation after counselling. The samples were collected over one year and followed up till delivery. AF was sent for QF-PCR study for chromosomes 13, 18, 21 and 23. There was no procedure related miscarriage, or IUFD. There were seventeen trisomy 21, two 47XXX and one 47XXY. All pregnancies with fetal aneuploidies underwent termination. All 552 women with normal report were followed up and none had IUFD or clinical features of trisomy 21 after term or preterm births. The current study stresses the clinical importance of shorter TAT advantage and the slight cost advantage of QF-PCR over FISH. The current study shows QF-PCR is a satisfactory method for diagnosing chromosomal anomaly from amniotic fluid in screen positive 
population, has advantages and might be preferred more in near future in place of FISH, especially in the scenario of increasing awareness of biochemical screening for chromosomal aneuploidies in India. DNA based test like QF-PCR has unlimited opportunity for testing for other disorders as well which may be of relevance in some cases However, more such studies with increased numbers and in multiple pregnancies are required. The current study is ongoing and hopefully we will be able to present such data soon.

\section{References}

1. Langlois S, Duncan A, Wilson RD, Audibert F, Brock JA, Carroll J, Cartier L, Désilets VA, Gagnon A, Johnson JA, Murphy-Kaulbeck L. Use of a DNA method, QFPCR, in the prenatal diagnosis of fetal aneuploidies. $J$ Obstet Gynaecol Canada. 2011;33,955-60.

2. Badenas C, Rodríguez-Revenga L, Morales C, Mediano C, Plaja A, Pérez-Iribarne MM, Soler A, Clusellas N, Borrell A, Sánchez MÁ, Miró E. “Assessment of QFPCR as the first approach in prenatal diagnosis. $J \mathrm{Mol}$ Diagn. 2010;12:828-34.

3. Feldman B, Ebrahim SA, Hazan SL, Gyi K, Johnson MP, Johnson A, Evans MI. Routine prenatal diagnosis of aneuploidy by FISH studies in high-risk pregnancies. American Journal of Medical Genetics Part A. 2000;90:233-8.

4. Badenas C, Rodríguez-Revenga L, Morales C, Mediano C, Plaja A, Pérez-Iribarne MM, Soler A, Clusellas N, Borrell A, Sánchez MÁ, Miró E. Assessment of QF-PCR as the first approach in prenatal diagnosis. The Journal of Molecular Diagnostics (2010) 12, 828-34.
5. Kong X, Li L, Sun L, Fu K, Long J, Weng X, Ye X, Liu $X$, Wang B, Yan S, Ye H. Rapid diagnosis of aneuploidy using segmental duplication quantitative fluorescent PCR. PloS one. (2014) 9, e88932.

6. Griffiths AJ. "An introduction to genetic analysis". Macmillan; 2005.

7. Nicolini U, Lalatta F, Natacci F, Curcio C, Bui TH. The introduction of QF-PCR in prenatal diagnosis of fetal aneuploidies: time for reconsideration. Human reproduction update. 2004;10,541-8.

8. de Moraes RW, de Carvalho MH, de Amorim-Filho AG, Francisco RP, Romão RM, Levi JE, Zugaib M. Validation of QF-PCR for prenatal diagnoses in a Brazilian population. Clinics. 2017;72, 400-4.

9. Srinivasan M, Sarita A. Genetic Characterization of microsatellite markers (D21S1413, D21S1412, D13S258 and D13S651) flanking 21st and 13th Chromosome: assessing applicability in aneuploidy diagnosis by QFPCR. Gene Therapy and Molecular Biology. 2014;16,190-4.

How to cite this article: Ghosh $\mathrm{K}$, Appaswamy G, Jayakrishna T. Quantitative fluorescent PCR (QF-PCR) for prenatal diagnosis of fetal aneuploidy: Indian experience. Ind $\mathrm{J}$ Obstet Gynecol Res. 2018;5(3):419-423. 